\title{
Structures of Metalloporphyrin-Oligomer Multianions: Cofacial versus Coplanar Motifs as Resolved by Ion Mobility Spectrometry
}

\author{
Katrina Brendle, ${ }^{\dagger}$ Ulrike Schwarz, ${ }^{\dagger}$ Patrick Jäger, ${ }^{\dagger}$ Patrick Weis, ${ }^{* \dagger}$ and Manfred Kappes ${ }^{*, \dagger, \ddagger}$ \\ ${ }^{\dagger}$ Institute of Physical Chemistry, Karlsruhe Institute of Technology, Fritz-Haber-Weg 2, 76131 Karlsruhe, Germany \\ ${ }^{\ddagger}$ Institute of Nanotechnology, Karlsruhe Institute of Technology, Hermann-von-Helmholtz-Platz 1, 76344 Eggenstein-Leopoldshafen, \\ Germany
}

Supporting Information

ABSTRACT: We have combined ion mobility mass spectrometry with quantum chemical calculations to investigate the gasphase structures of multiply negatively charged oligomers of mesotetra(4-sulfonatophenyl)metalloporphyrins comprising the divalent metal centers $\mathrm{Zn}^{\mathrm{II}}, \mathrm{Cu}^{\mathrm{II}}$, and $\mathrm{Pd}^{\mathrm{II}}$. Sets of candidate structures were obtained by geometry optimizations based on calculations at both the semiempirical PM7 and density functional theory (DFT)

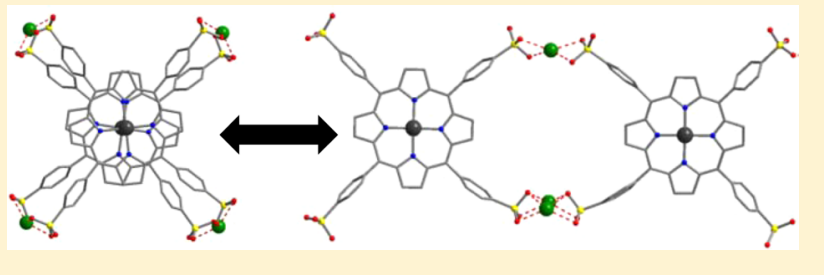
levels. The corresponding theoretical cross sections were calculated with the projection approximation and also with the trajectory method. By comparing these collision cross sections with the respective experimental values we were able to assign oligomer structures up to the tetramer. In most cases the cross sections of the lowest energy isomers predicted by theory were found to agree with the measurements to within the experimental uncertainty (2\%). Specifically, we find that for a given oligomer size the structures are independent of the metal center but depend strongly on the charge state. Oligomers in low charge states with a correspondingly larger number of sodium counterions tend to form stacked, cofacial structures reminiscent of $\mathrm{H}$-aggregate motifs observed in solution. By contrast, in higher charge states, the stack opens to form coplanar structures.

\section{INTRODUCTION}

Metalloporphyrins constitute the reactive centers in a large number of biochemical systems such as hemoglobin, myoglobin, and chlorophyll. As a consequence, they are wellstudied in condensed phase. ${ }^{1}$ It is also well-known that porphyrins and metalloporphyrins can self-assemble into larger oligomers in solution. This aggregation has attracted considerable interest, especially for porphyrins functionalized with highly polar groups such as sulfonic acids. ${ }^{2-9}$ Much of this effort has been focused on water-soluble meso-tetra(4sulfonatophenyl)porphyrin (TPPS). Ribó et al. ${ }^{6}$ investigated aqueous solutions thereof spectroscopically. In studies performed at various concentrations and $\mathrm{pH}$ values, they found evidence for the formation of small $\mathrm{J}$ (edge-to-edge) and $\mathrm{H}$ (face-to-face) aggregates. The light scattering measurements of Micali et al. ${ }^{10}$ have in fact suggested that even largermesoscopic-self-similar clusters of TPPS J-aggregates may form in solution. More recently, Hollingsworth et al. ${ }^{9}$ have investigated the aggregation of TPPS in aqueous solution using $\mathrm{UV}$-vis and fluorescence spectroscopy, small-angle X-ray scattering, analytical ultracentrifugation, and transmission electron microscopy. They inferred stacks of ring structures containing typically 25 monomeric units.

TPPS oligomers as well as aggregates of the corresponding metalloporphyrins, MTPPS, can also be prepared and studied in gas phase, i.e., in the absence of solvent molecules. For example, multiply negatively charged aggregates of MTPPS (also containing sodium counterions) can be generated by electrospray ionization (ESI) and detected with mass spectrometry. To what extent such isolated aggregates can also provide an indirect measure of aggregation in solution is presently an interesting unresolved issue. In previous studies, we have investigated the structures, relative stabilities, and photoelectron spectra of isolated multianionic MTPPS dimers and trimers comprising trivalent metal centers, $\mathrm{M}^{\mathrm{III}}=\mathrm{Mn}^{3+}$ and $\mathrm{Fe}^{3+}$ (with an initial focus on homometallic oligomers). The observed daisy-chain-like structures are primarily determined by (partially ionic) bonds between the $\mathrm{M}^{\mathrm{III}}$ center of one porphyrin subunit and one of the sulfonic acid groups of another porphyrin. ${ }^{11,12}$ Multianionic aggregates of $\mathrm{M}^{\mathrm{III}} \mathrm{TPPS}$, therefore, do not form (or do not retain) small $\mathrm{H}$ - or $\mathrm{J}$ aggregate-like motifs under isolated solvent-free conditions.

In the present study we revisit this issue-now focusing on the structures of MTPPS oligomers comprising a variety of different divalent metal centers, $\mathrm{M}^{\mathrm{II}}=\mathrm{Zn}^{2+}, \mathrm{Cu}^{2+}$, and $\mathrm{Pd}^{2+}$. The corresponding structures were determined using a combination of ion mobility mass spectrometry ${ }^{13,14}$ (IMSMS) with quantum chemical calculations. This has proven to be a powerful approach to structurally characterize many classes of large molecular ions in gas phase such as DNA oligonucleotides, $^{15}$ peptides, ${ }^{16-18}$ and numerous metal-organic species. ${ }^{11,19}$ Here we apply it to small oligomers (up to tetramers)

Received: August 10, 2016

Revised: October 11, 2016

Published: October 11, 2016 
of $\mathrm{M}^{\mathrm{II}} \mathrm{TPPS}$. In particular, we have compared their experimental collision cross sections to quantum chemically derived model structures using both the projection approximation and the trajectory method. For the highly negatively charged species of interest here, which often comprise hundreds of atoms, the models were obtained by geometry optimizations at the PM7 level [as validated by density functional theory (DFT) calculations].

In contrast to $\mathrm{M}^{\mathrm{III}} \mathrm{TPPS}$, we find that $\mathrm{M}^{\mathrm{II}} \mathrm{TPPS}$ can form low negative charge state oligomers whose stacked structures resemble $\mathrm{H}$-aggregate-like motifs. Increasing the negative charge state by reducing the number of complexed counterions leads to coplanar geometries.

\section{EXPERIMENTAL METHODS}

$\mathrm{M}^{\mathrm{II}}$ meso-tetra(4-sulfonatophenyl)porphyrin $(\mathrm{M}=\mathrm{Zn}, \mathrm{Cu}, \mathrm{Pd})$ were obtained as sodium salts from Frontier Scientific and used without further purification. For electrospray ionization we used concentrations of $1-3 \mathrm{mmol} / \mathrm{L}$ in water/methanol in all cases. High-resolution mass spectra were acquired on a LTQ Orbitrap XL instrument (Thermo Fisher). Collision cross sections were obtained in a home-built instrument that comprises an electrospray ion source (similar to the one used in the LTQ instrument), an ion mobility drift cell filled with 23 mbar of helium as buffer gas (monitored by two MKS capacitance manometers), two quadrupole mass spectrometers (Extrel), and a time-of-flight electron spectrometer. Details of the setup are described elsewhere. ${ }^{20}$ Briefly, the ions emerging from the transfer capillary of the electrospray source are stored in an electrodynamic (entrance) ion funnel, subsequently injected into the drift cell, and guided by a static, homogeneous electric field $(\approx 10 \mathrm{~V} / \mathrm{cm})$ to the exit funnel that also serves as pressure-limiting aperture to the high-vacuum part of the instrument. After passing the funnel the ions are mass selected in the first quadrupole mass filter (set to a resolution $m / \Delta m \approx$ 100; for the experiments presented here, the second quadrupole is operated in rf-only mode) and their arrival time is recorded by an oscilloscope (TDS2022, Tektronics). Under the low-field conditions that we operate at, the arrival time depends linearly on ratio of buffer gas pressure $(p)$ and drift field $(E)$, the slope being proportional to the ion mobility. Typically between 8 and 10 arrival time measurements at different $p / E$ ratios are used to extract an ion mobility value. The ion mobility $K$ is closely related to the collision cross section $\Omega:^{21}$

$$
K=\frac{3 q}{16 N} \sqrt{\frac{2 \pi}{\mu k_{\mathrm{B}} T}} \frac{1}{\Omega}
$$

$N$ and $T$ are the number density and temperature of the buffer gas, $q$ is the ion charge, and $\mu$ is the reduced mass of ion and buffer gas (helium). For each species we measured the collision cross section at least 10 times on at least three different days. Under the conditions of these experiments the ion mobility resolution was 25 (fwhm). The typical error in resulting collision cross sections was $\pm 2 \%$. We attribute the peak tailing commonly observed in our ion arrival time distributions to field inhomogeneities in the exit ion funnel because the shape of this tail depends strongly on the rf and dc voltages applied to it. In order to assign structures, the experimental collision cross sections have been compared with candidate structures based on quantum chemical calculations.

\section{COMPUTATIONAL METHODS}

The search for candidate structures has been performed with the semiempirical PM7 method as implemented in MOPAC $12 .^{22}$ With this comparatively fast method it is possible to explore a large number of candidate structures, even for the tetramers which comprise more than 350 atoms. We validated the method for monomers and dimers by comparing with DFT calculations as implemented in the TURBOMOLE package ${ }^{23}$ with the $B P 86^{24,25}$ functional and two basis sets: the comparatively small def-SV(P) basis $\operatorname{set}^{26}$ and the much larger TZVPP basis set. For this we focused primarily on zinccontaining porphyrins since the central $\mathrm{Zn}$ (II) has a closed shell $\left(\mathrm{d}^{10}\right)$ electronic configuration, which facilitates the DFT calculations. Candidate structures were confirmed or ruled out by comparing their calculated collision cross sections with the respective experimental values. The corresponding collision cross sections were calculated with two methods: the projection approximation (PA) ${ }^{27}$ and the trajectory method (TM), ${ }^{28}$ both implemented in the MOBCAL package. ${ }^{29}$

\section{RESULTS AND DISCUSSION}

Monomer Tetraanions as Calibration Points. Under our ESI conditions the resulting anion mass distribution was dominated by the monomeric tetraanions $[\mathrm{MTPPS}]^{4-}$, see Figure 1 and Supporting Information. On the basis of their

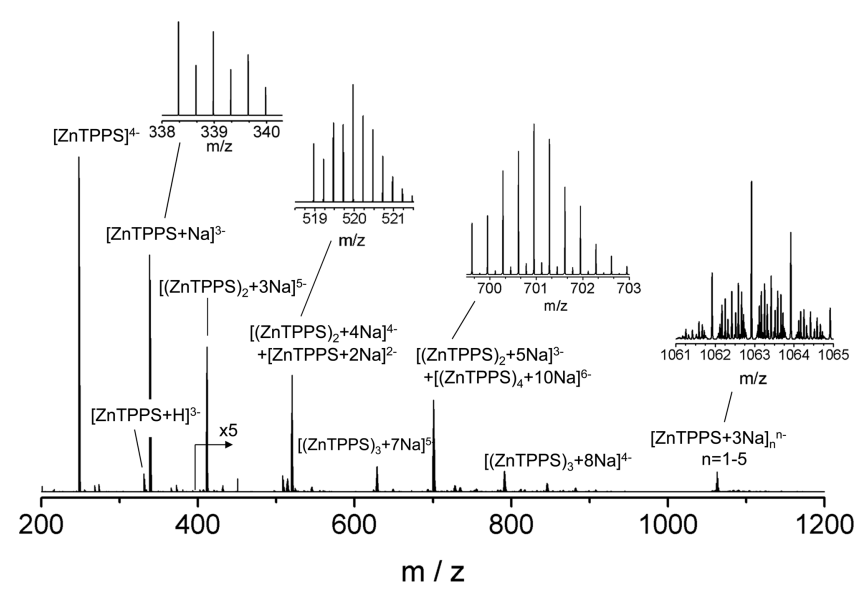

Figure 1. ESI mass spectrum of $\mathrm{Zn}$ (II) meso-tetra(4-sulfonatophenyl)porphyrin (sodium salt), as recorded by the LTQ Orbitrap XL instrument $(1 \mathrm{mmol} / \mathrm{L}$ solution in water/methanol). On the basis of the isotope pattern we can differentiate between monomers and oligomers of equal $\mathrm{m} / z$ ratio: the doubly charged monomer [ZnTPPS $+2 \mathrm{Na}]^{2-}$ and its dimer $\left[(\mathrm{ZnTPPS})_{2}+4 \mathrm{Na}\right]^{4-}$ are present in similar amounts; the dimer $\left[(\mathrm{ZnTPPS})_{2}+2 \mathrm{Na}\right]^{6-}$ corresponding to the triply charged monomer, [ZnTPPS $+\mathrm{Na}]^{3-}$, is absent. At $\mathrm{m} / z=1063$, we also observe oligomers up to the pentamer in the corresponding charge states (i.e., $[\mathrm{ZnTPPS}+3 \mathrm{Na}]_{n}{ }^{n-}, n=1-5$ ), in addition to the expected singly charged monomer.

arrival time distributions (see Figure 2 and Supporting Information) we determined the cross sections for the quadruply charged monomers to be $[\mathrm{ZnTPPS}]^{4-} 257 \AA^{2}$, [CuTPPS $]^{4-} 256 \AA^{2}$, and [PdTPPS] ${ }^{4-} 256 \AA^{2}$ (see Table 1). Note that they agree with each other to within $1 \%$-reflecting the fact that for the metals probed the central atom has no significant influence on the overall metalloporphyrin structure. By comparison, the calculated collision cross sections based on the hard-spheres projection approximation, PA-CCS (with the hard-spheres radii as implemented in the MOBCAL package, 

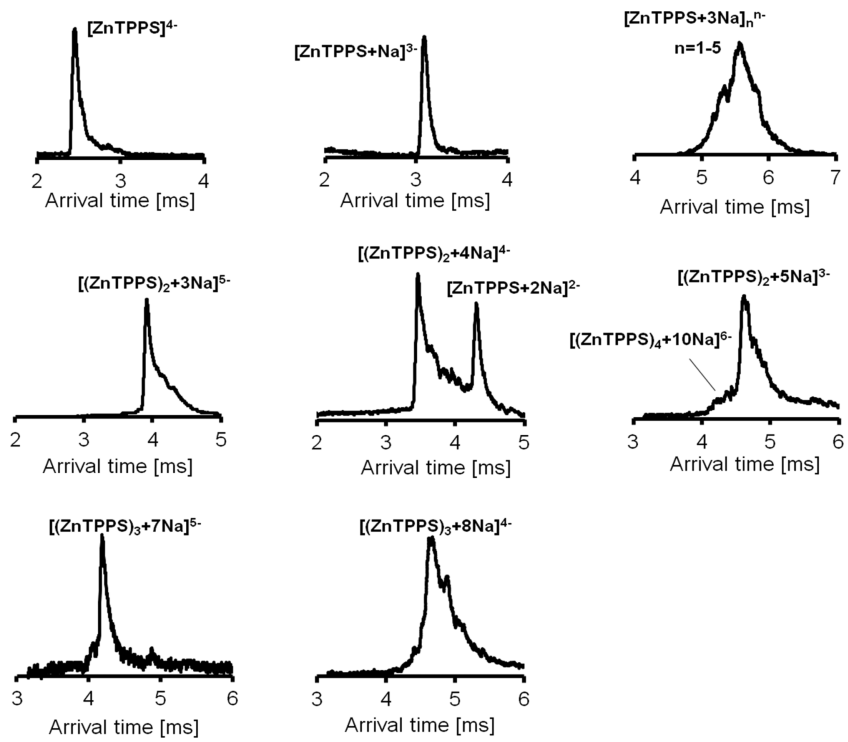

Figure 2. Typical arrival time distributions of ZnTPPS oligomers. The tailing behind each peak is a consequence of the ion funnel at the exit of the drift cell, i.e., it represents an experimental artifact.

Table 1. Experimental Collision Cross Sections

\begin{tabular}{lccc} 
& $\mathrm{M}=\mathrm{Zn}\left(\AA^{2}\right)$ & $\mathrm{M}=\mathrm{Pd}\left(\AA^{2}\right)$ & $\mathrm{M}=\mathrm{Cu}\left(\AA^{2}\right)$ \\
$\mathrm{MTPPS}^{4-}$ & Monomers & & \\
{$[\mathrm{MTPPS}+\mathrm{H}]^{3-}$} & $257 \pm 5$ & $256 \pm 5$ & $256 \pm 5$ \\
{$[\mathrm{MTPPS}+\mathrm{Na}]^{3-}$} & $257 \pm 5$ & $255 \pm 5$ & $256 \pm 5$ \\
& $253 \pm 5$ & $251 \pm 5$ & $249 \pm 5$ \\
{$\left[(\mathrm{MTPPS})_{2}+3 \mathrm{Na}\right]^{5-}$} & $476 \pm 10$ & $478 \pm 10$ & $472 \pm 10$ \\
{$\left[(\mathrm{MTPPS})_{2}+4 \mathrm{Na}\right]^{4-}$} & $370 \pm 7$ & $378 \pm 8$ & $372 \pm 7$ \\
{$\left[(\mathrm{MTPPS})_{2}+5 \mathrm{Na}\right]^{3-}$} & $359 \pm 7$ & $367 \pm 7$ & $368 \pm 7$ \\
& Trimers & & $(1) 587 \pm 12$ \\
{$\left[(\mathrm{MTPPS})_{3}+7 \mathrm{Na}\right]^{5-}$} & $581 \pm 12$ & $573 \pm 11$ & $(2) 563 \pm 11$ \\
{$\left[(\mathrm{MTPPS})_{3}+8 \mathrm{Na}\right]^{4-}$} & $477 \pm 10$ & $484 \pm 10$ & $479 \pm 10$ \\
{$\left[(\mathrm{MTPPS})_{4}+10 \mathrm{Na}\right]^{6-}$} & $661 \pm 35$ & $676 \pm 35$ & $661 \pm 35$ \\
\hline
\end{tabular}

i.e., $2.20 \AA$ for $\mathrm{H}, 2.70 \AA$ for $\mathrm{C}, \mathrm{N}$, and $\mathrm{O}, 3.50 \AA$ for the heavier atoms) for the PM7-optimized structures turn out to be $249 \AA^{2}$, i.e., systematically $3 \%$ smaller than the experimental value. This is within the expected deviation between experiment and theory (around 3\%) due to statistical (1-2\%) and systematic experimental errors ( $1 \%$, due to uncertainties in pressure, temperature, and effective electrical field plus drift length). A further source of error is the precision of the geometry optimization at PM7 level. Note that the diameter of the TPPS molecule, calculated as the distance between diagonal sulfur atoms (19.04 $\AA$ ), is roughly $2 \%$ smaller than the $S-S$ distance calculated at DFT level (19.38 $\AA$, BP86, def-SV(P)). As a consequence, the DFT-based collision cross section $\left(253 \AA^{2}\right)$ agrees with experiment to within $1.5 \%$. A more substantial source of error is the projection approximation itself: The PACCS of a molecule is determined by its geometrical shape only; the model completely neglects the attractive charge-induced dipole interaction between the molecular ion and the helium buffer gas. Furthermore, the parameters (hard-sphere radii) have been calibrated against ion mobility data on cations, mostly oligopeptides. In light of these various, rather large sources of error, the observed $1.5-3 \%$ difference between theory (DFT, PM7) and experiment is surprisingly good-but might also be due to a fortuitous cancellation of errors.

The trajectory method (TM) for calculating collision cross sections is by far more sophisticated than the PA-CCS approach, since it takes both short-range repulsive and longrange attractive interactions into account. Within the TM method the collision cross section is not approximated by a projected area but instead calculated by integration of the buffer gas-molecular ion trajectories. In order to do so, TM treats the interaction of the molecular ion with the buffer gas (helium in our case) by a sum of Lennard-Jones-type interactions between each atom of the molecular ion and helium with element specific Lennard-Jones parameters $r_{0}$ (distance) and $\varepsilon$ (energy depth). Furthermore, it includes the ion-induced dipole interaction by means of a sum of $q_{i}^{2} / r_{i}^{4}$ terms, with the sum running over all constituent atoms, $q_{i}$ being the partial charge on atom $i$ and $r_{i}$ its actual distance to the scattering helium atom. This attractive interaction scales with the polarizability of helium and gains importance for highly charged ions. For the TPPS monomer tetraanions we assume that each of the 12 oxygen atoms carries a charge of $-1 / 3$; all other atoms remain uncharged. With this assumption and the parameters implemented in MOBCAL we obtain a TM-CCS of $271 \AA^{2}$ (PM7), i.e., systematically 5\% larger than the experimental values and $8 \%$ larger than the PA-CCS. This deviation is too large for precise structure assignments. A possible reason is that the element-specific Lennard-Jones radii implemented in the MOBCAL package, that have been calibrated mostly for cationic systems, $^{28,29}$ are slightly too large. Since the structure of the monomeric metal-porphyrin tetraanion [MTPPS $]^{4-}$ is well-known (metal center in the plane of the porphyrin ring, phenyl groups rotated out of porphyrin plane due to steric hindrance), ${ }^{30,31}$ we use the average experimental CCS $\left(256 \AA^{2}\right)$ as calibration point and scale all TM atomic radii (strictly speaking the parameter $r_{0}$ of the Lennard-Jones potential of each element with helium) by roughly 0.92 . Specifically we use $2.19 \AA$ for hydrogen, $2.80 \AA$ for carbon, nitrogen, oxygen, and sodium, and $3.22 \AA$ for sulfur and the transition metals. For the sake of consistency we scale the PA hard-spheres radii as well, by roughly 1.02 to match the experimental cross sections $(2.28$ $\AA$ for $\mathrm{H}, 2.80 \AA$ for $\mathrm{C}, \mathrm{N}, \mathrm{Na}$, and $\mathrm{O}, 3.62 \AA$ for $\mathrm{S}$ and the transition metals). With these calibrated radii we reproduce the experimental CCS of the monomer tetraanions with both the projection approximation and trajectory method (and in each case the PM7 structural model) to within $1 \AA^{2}$. Note that we use the PM7-optimized geometry instead of the DFToptimized geometry for calibration in order to be able to assign structures not only for monomers, but also for dimers, trimers, and tetramers with one atomic parameter set (DFT is not feasible for the trimers and tetramers due to the large number of atoms in these systems).

Compared to the quadruply charged monomers $[\mathrm{MTPPS}]^{4-}$, the sodiated triply charged monomers $[\mathrm{MTPPS}+\mathrm{Na}]^{3-}$ have slightly smaller collision cross sections $\left(253,249\right.$, and $251 \AA^{2}$ for $\mathrm{M}^{\mathrm{II}}=\mathrm{Zn}^{2+}, \mathrm{Cu}^{2+}$, and $\mathrm{Pd}^{2+}$, respectively). This is surprising at first glance since the additional atom increases the geometrical cross section (according to the PM7-optimized $[\mathrm{ZnTPPS}+\mathrm{Na}]^{3-}$ structure, the sodium atom is in close proximity to one of the sulfonyl groups, leaving the rest of the molecule unchanged). Consequently, the PA-CCS increases upon sodiation by $2.5 \%$ from $256 \AA^{2}$ for [MTPPS $]^{4-}$ to $263 \AA^{2}$ for $[\mathrm{MTPPS}+\mathrm{Na}]^{3-}$. This is $4 \%$ larger than the experimental 
value of $253 \AA^{2}$. This implies that the experimentally observed CCS decrease might be a consequence of the different charge distributions in tetra- and trianions. Calculating the TM-CCS with the assumption that the sodium atom carries a full positive charge $(+1)$ and that each oxygen of the sulfonic acid groups has a negative charge of $(-1 / 3)$ results in a value of $260 \AA^{2}$ for the triply charged monomer, which is in better agreement with experiment than the PA-CCS value, now only $2.5 \%$ too large. Assuming that the sodium completely neutralizes a sulfonic acid group (i.e., zero charge on sodium and on three of the oxygens) results in a CCS of $256 \AA^{2}$ which is within (1\%) of the experimental value. By comparing PA- and TM-CCS it becomes clear that for accurate CCS calculations the charge distribution has to be taken into account.

Dimers. As can be seen in the mass spectrum shown in Figure 1, we observe dimers in four different charge statesfrom doubly up to quintuply charged. At $m / z=1063$, the mass to charge ratio corresponding to the doubly charged dimer, we in fact observe a mixture of the singly charged monomer, the doubly charged dimer, and the corresponding oligomers up to the pentamer (see Figure 1). The corresponding arrival time distribution (ATD) is very broad (see Figure 2 and Supporting Information), and it is not possible to determine accurate collision cross sections. We therefore concentrate in the following on triply, quadruply, and quintuply charged dimers.

$\left[(\mathrm{MTPPS})_{2}+5 \mathrm{Na}\right]^{3-}$. At $\mathrm{m} / z=701$ we observe both the triply charged dimers $\left[(\mathrm{MTPPS})_{2}+5 \mathrm{Na}\right]^{3-}$ and at a much lower intensity the sixfold-charged tetramers (see Figure 1). The corresponding arrival time distribution is bimodal; the small leading peak corresponds to the tetramer (see Figure 2 and Supporting Information), the main peak to the dimer. By following the dependence of this peak on different pressure-tovoltage ratios we determine CCS values of $359 \AA^{2}(\mathrm{Zn}), 366 \AA^{2}$ (Pd), and $368 \AA^{2}(\mathrm{Cu})$ for the triply charged dimers. The differences between metals are within the experimental uncertainty of $2 \%$. In order to determine the structure of $\left[(\mathrm{MTPPS})_{2}+5 \mathrm{Na}\right]^{3-}$ we optimized a series of 20 candidate structures at PM7 and DFT level. The lowest energy structures obtained are summarized in Figure 3 and Table 2 (see

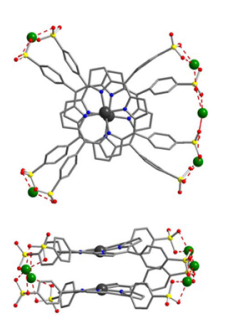

(I) $\triangle E_{P_{m} 7} \quad 0 \mathrm{eV}$ TM-CCS $359 \AA^{2}$ PA-CCS $340 \AA^{2}$
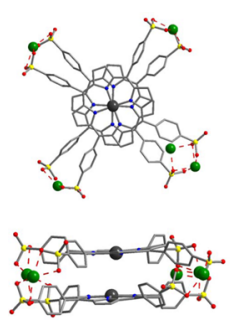

$$
\begin{gathered}
\text { (II) } \triangle E_{P_{m 7}} \quad 0.23 \mathrm{eV} \\
\text { TM-CCS } 361 \AA^{2}
\end{gathered}
$$$$
\text { PA-CCS } 344 \AA^{2}
$$
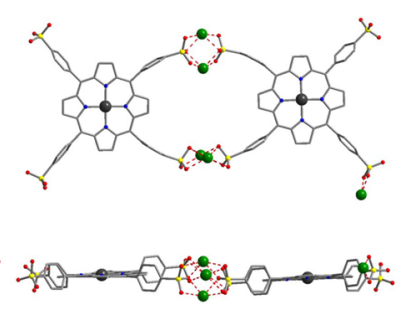

(III) $\Delta \mathrm{E}_{\mathrm{Pm} 7} \quad 2.43 \mathrm{eV}$

TM-CCS $477 \AA^{2}$

PA-CCS $468 \AA^{2}$
Figure 3. Low-energy structures of $\left[(\mathrm{ZnTPPS})_{2}+5 \mathrm{Na}\right]^{3-}$ : relative energies and collision cross sections [Zn (gray), $\mathrm{Na}$ (green), $\mathrm{O}$ (red), $\mathrm{N}$ (blue), and $\mathrm{S}$ (yellow)]. The experimental collision cross section is $359 \AA^{2}$.

Supporting Information for a more complete list). Both PM7 and DFT predict the same lowest energy structure (structure I), which corresponds to a stack (face-to-face or H-aggregate) of the two porphyrin rings. The sodium cations interact with the anionic sulfonic acid groups of both rings and thereby serve as a "glue" to hold the stacked geometry together. The central
Table 2. Relative Energies for the Three Lowest Energy Isomers of $\left[(\mathrm{ZnTPPS})_{2}+5 \mathrm{Na}\right]^{3-a}$

$\begin{array}{llcc} & \begin{array}{c}\text { PM7 } \\ (\mathrm{eV})\end{array} & \begin{array}{c}\text { DFT (BP86, def-SV(P)) } \\ (\mathrm{eV})\end{array} & \begin{array}{c}\text { DFT (BP86, TZVPP) } \\ (\mathrm{eV})\end{array} \\ \text { I } & 0 & 0 & 0 \\ \text { II } & 0.23 & 0.48 & 0.43 \\ \text { III } & 2.43 & 3.43 & 2.84\end{array}$

${ }^{a}$ As shown in Figure 3 and as calculated with different methods.

metal atoms play a minor role. Their PM7 distance is $3.8 \AA$, which is comparable with the separations in covalently interlinked cofacial bisporphyrins as prepared by chemical synthesis in solution (3.87-4.80 $\AA$, depending on linker). ${ }^{32}$ Structure II represents a variant of this motif. The difference is that in structure II the average sodium-sodium distance is slightly larger (in structure II two of the sodium atoms interact with a pair of sulfonic acid groups, while in structure I four of the sulfonic acid groups interact with three sodium atoms, i.e., the charge is better delocalized). As a consequence, structure II is higher in energy by $0.23 \mathrm{eV}$ according to PM7. At the DFT level the energy difference is even larger (def-SV $(\mathrm{P}), 0.48 \mathrm{eV}$; TZVPP, $0.43 \mathrm{eV}$ ), depending on the basis set used. The TMCCS of these structures are basically identical (359 and $361 \AA^{2}$ ) and in perfect agreement with the experimental value of $359 \AA^{2}$. On the basis of the ion mobility we cannot distinguish between the two structures. We can, however, rule out structures that deviate significantly from the face-to-face orientation. An example is structure III, where the two porphyrin rings are in the same plane [side-by-side or J-aggregate, $\mathrm{Zn}-\mathrm{Zn}$ distance $19.8 \AA$ (PM7)]. The TM-CCS of structure III is $477 \AA^{2}, 25 \%$ larger than the experimental value. Furthermore, its energy is much higher than that of structure I: $2.43 \mathrm{eV}$ according to PM7 and $3.43 \mathrm{eV}$ according to DFT. So both PM7 and DFT clearly favor the structure I that agrees with the experimental cross section. It is interesting to note that the PA-CCS for structure I is $5 \%$ smaller than the TM-CCS and the experimental value (note that we calibrated the atomic parameters for the monomer!). Since structure $\mathrm{I}$ is the most compact structure possible (see also Supporting Information) it is clear that this deviation is a consequence of the assumptions inherent to the projection approximation.

$\left[(\mathrm{MTPPS})_{2}+4 \mathrm{Na}\right]^{4-}$. The cross section measurement of the quadruply charged dimers $\left[(\mathrm{MTPPS})_{2}+4 \mathrm{Na}\right]^{4-}$ is complicated by the fact that the doubly charged monomer [MTPPS + $2 \mathrm{Na}]^{2-}$ has the same mass-to-charge ratio, and therefore, these species cannot be resolved in the quadrupole mass spectrometer (at the typical experimental resolution $\approx 100$ ). However, in the corresponding arrival time distribution we observe two peaks (see Figure 2 and Supporting Information), the first of which we assign to the dimer, the second to the monomer (see above). Using this discrimination procedure, the cross sections of the quadruply charged dimers of $\mathrm{M}=\mathrm{Zn}, \mathrm{Cu}$, and Pd can be determined as 370,372 , and $378 \AA^{2}$, respectively, i.e., they are identical within the experimental error. The energetically favored $\left[(\mathrm{ZnTPPS})_{2}+4 \mathrm{Na}\right]^{4-}$ structure at both PM7 and DFT level is again a face-to-face structure; each of the four sodium ions connects two opposing sulfonic acid groups, see Figure 4 and Table 3 . The TM-CCS is $368 \AA^{2}$, in agreement with experiment within 1\%. PA-CCS is again 5\% smaller, 349 $\AA^{2}$. Rotating the porphyrin rings with respect to each other while maintaining their cofacial arrangement (structure II) reduces the number of ionic interactions and thereby increases 

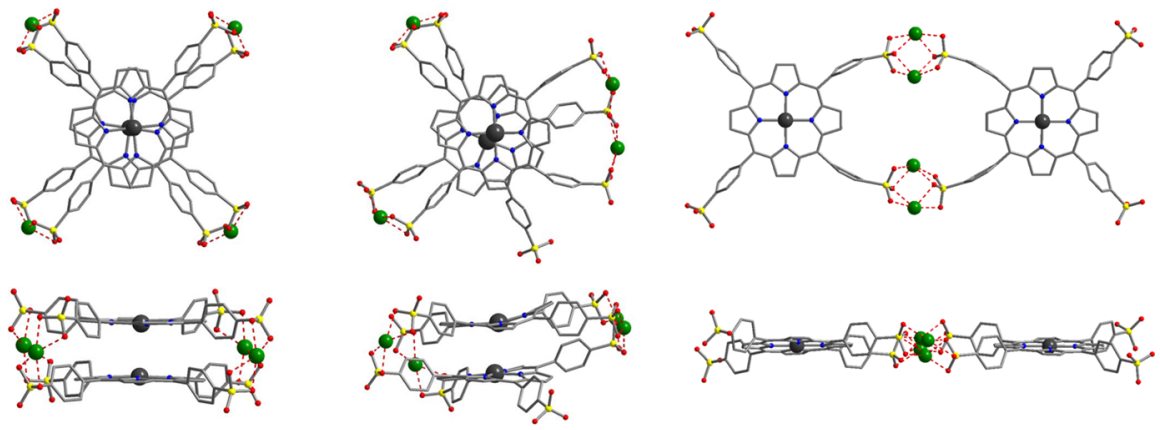

(I) $\Delta \mathrm{E}_{\mathrm{Pm} 7} \quad 0 \mathrm{eV}$
$\mathrm{TM}-\mathrm{CCS} 368 \AA^{2}$
$\mathrm{PA}-\mathrm{CCS} 349 \AA^{2}$
(II) $\Delta \mathrm{E}_{\mathrm{Pm} 7} \quad 0.95 \mathrm{eV}$
TM-CCS $369 \AA^{2}$
(III) $\Delta \mathrm{E}_{\mathrm{Pm} 7} \quad 1.27 \mathrm{eV}$
TM-CCS $469 \AA^{2}$
PA-CCS $463 \AA^{2}$

Figure 4. Low-energy structures of $\left[(\mathrm{ZnTPPS})_{2}+4 \mathrm{Na}\right]^{4-}$ : relative energies and collision cross sections. The experimental collision cross section is $368 \AA^{2}$.

Table 3. Relative Energies for the Three Lowest Energy Isomers of $\left[(\mathrm{ZnTPPS})_{2}+4 \mathrm{Na}\right]^{4-a}$

$\begin{array}{llcc} & \begin{array}{c}\text { PM7 } \\ (\mathrm{eV})\end{array} & \begin{array}{c}\text { DFT (BP86, def-SV(P)) } \\ (\mathrm{eV})\end{array} & \begin{array}{c}\text { DFT (BP86, TZVPP) } \\ (\mathrm{eV})\end{array} \\ \mathrm{I} & 0 & 0 & 0 \\ \text { II } & 0.95 & 0.99 & 0.88 \\ \text { III } & 1.27 & 2.59 & 2.01\end{array}$

${ }^{a}$ As shown in Figure 4 and as calculated with different methods.

the energy by almost $1 \mathrm{eV}$ (PM7, DFT). The CCS remains basically unchanged. Again, the side-by-side motif (structure III) can be clearly ruled out: according to PM7, it is $1.27 \mathrm{eV}$ higher in energy and has a much larger TM-CCS of $469 \AA^{2}$, $27 \%$ above the experimental value (PA-CCS $463 \AA^{2}, 26 \%$ above the experimental value). At the DFT (BP86, def-SV(P) basis) level, the energy difference is even larger, 2.59 and $2.01 \mathrm{eV}$, depending on the basis set. So it is clear that in both the triply and the quadruply charged dimers the porphyrin subunits are stacked in a face-to-face arrangement.

$\left[(\mathrm{MTPPS})_{2}+3 \mathrm{Na}\right]^{5-}$. For the quintuply charged dimers $\left[(\mathrm{MTPPS})_{2}+3 \mathrm{Na}\right]^{5-}$ we measure a CCS of $476 \AA^{2}$ for $\mathrm{M}=$ $\mathrm{Zn}, 478 \AA^{2}$ for $\mathrm{M}=\mathrm{Pd}$, and $472 \AA^{2}$ for $\mathrm{M}=\mathrm{Cu}$. Again, the CCS is independent of the metal center to within the experimental error. As before, we calculate candidate structures by geometry optimization of ca. 25 different starting geometries. With PM7 we find a quasi-planar side-by-side structure (structure I, see Table 4 and Figure 5) to be lowest in energy (see Supporting Information for a more complete list of candidate structures). This structure consists of two coplanar monomer units connected to each other by way of two pairs of sulfonic acid groups interconnected by three (two plus one) sodium counterions. The TM-CCS of this structure is $477 \AA^{2}$, in

Table 4. Relative Energies for the Four Lowest Energy Isomers of $\left[(\mathrm{ZnTPPS})_{2}+3 \mathrm{Na}\right]^{5-a}$

$\begin{array}{llcc} & \begin{array}{c}\text { PM7 } \\ (\mathrm{eV})\end{array} & \begin{array}{c}\text { DFT (BP86, def-SV(P)) } \\ (\mathrm{eV})\end{array} & \begin{array}{c}\text { DFT (BP86, TZVPP) } \\ (\mathrm{eV})\end{array} \\ \mathrm{I} & 0 & 0.36 & 0.17 \\ \text { II } & 0.32 & 0.23 & 0 \\ \text { III } & 0.95 & 0 & 0.01 \\ \text { IV } & 1.19 & 1.76 & 1.32\end{array}$

${ }^{a}$ As shown in Figure 5 and as calculated with different methods.
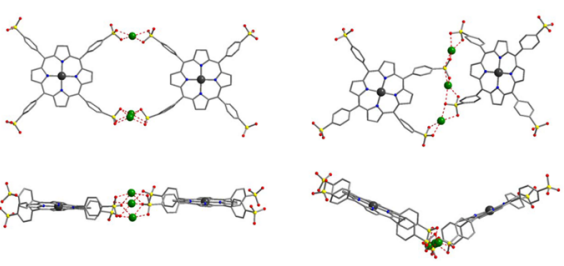
(I) $\triangle \mathrm{E}_{\mathrm{Pm} 7} \quad 0 \mathrm{eV}$ TM-CCS $477 \AA^{2}$ PA-CCS $468 \AA^{2}$
(II) $\triangle \mathrm{E}_{\mathrm{Pm} 7} \quad 0.32 \mathrm{eV}$ TM-CCS $459 \AA^{2}$ PA-CCS $442 \AA^{2}$

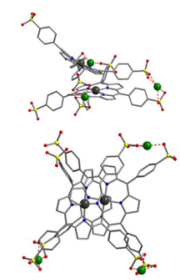

(III) $\Delta \mathrm{E}_{\mathrm{Pm} 7} \quad 0.95 \mathrm{eV}$ TM-CCS $397 \AA^{2}$ PA-CCS $371 \AA^{2}$

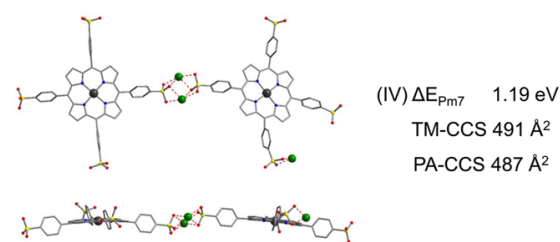

Figure 5. Low-energy structures of $\left[(\mathrm{ZnTPPS})_{2}+3 \mathrm{Na}\right]^{5-}$ : relative energies and collision cross sections. The experimental collision cross section is $476 \AA^{2}$.

almost perfect agreement with the experimental value (within $1 \%)$. The PA-CCS is again slightly smaller, $468 \AA^{2}$. A closely related, slightly tilted isomer (structure II) is $0.32 \mathrm{eV}$ higher in energy. It has a smaller TM-CCS section of $459 \AA^{2}$ (PA-CCS $442 \AA^{2}$ ), $4 \%$ below the experimental value-somewhat too close to rule it out completely. A stacked structure (III) with the two porphyrin rings basically facing each other is $0.95 \mathrm{eV}$ higher in energy. This structure is closely related to the favored structure for the lower charge states (see above). For $\left[(\mathrm{MTPPS})_{2}+3 \mathrm{Na}\right]^{5-}$ the stacking is not perfect since the three sodium counterions cannot connect all sulfonic acid groups of the two opposing porphyrins. This structure has a TM-CCS of $397 \AA^{2}$ (PA-CCS, $371 \AA^{2}$ ) and can be clearly ruled out on the basis of the collision cross section (17\% below the experimental value). It is interesting to note that a geometry optimization at the DFT level using a def-SV(P) basis set predicts this structure to be lowest in energy. This is, however, in strong contrast to the experimental findings: we clearly observe structure I (or variants thereof, such as structure II). A possible reason for the obvious failure of this method is a basis set superposition error. In order to estimate the magnitude of this effect we repeated the calculation using a much larger basis 

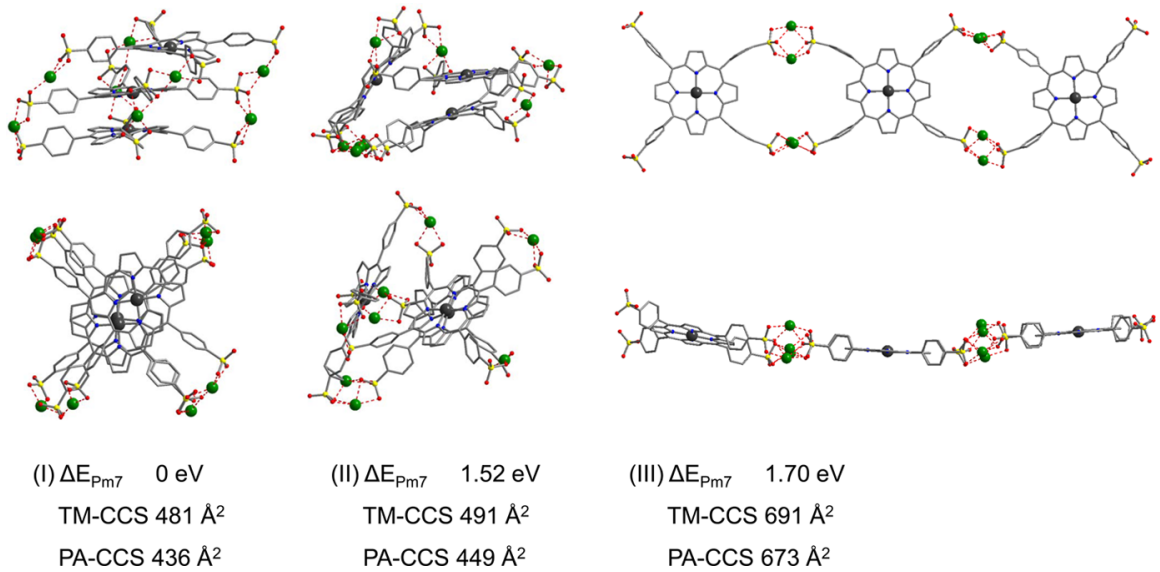
(II) $\Delta E_{P m 7} \quad 1.52 \mathrm{eV}$
TM-CCS $491 \AA^{2}$

(III) $\triangle \mathrm{E}_{\mathrm{Pm} 7} \quad 1.70 \mathrm{eV}$

TM-CCS $691 \AA^{2}$

PA-CCS $673 \AA^{2}$

Figure 6. Low-energy structures of $\left[(\mathrm{ZnTPPS})_{3}+8 \mathrm{Na}\right]^{4-}$ : relative energies and collision cross sections. The experimental collision cross section is $477 \AA^{2}$.

set. With the TZVPP-basis set, structure III becomes energetically degenerate with structure II. Since structure I is only $0.17 \mathrm{eV}$ higher it is impossible to assign a structure on the basis of the DFT energy alone (see Table 4). On the basis of the cross section it is, however, clear that structure I, the structure favored by PM7, is in best agreement with experiment. Note that a coplanar dimer (structure IV) connected via a single pair of phenyl sulfonic acid groups (plus sodium counterions) is more than $1 \mathrm{eV}$ higher in energy, independent of the method and basis set used. Its cross section (TM-CCS $491 \AA^{2}$, PA $487 \AA^{2}$ ) is similar to that of the preferred isomer $\mathrm{I}$, but based on the large energy difference we can rule out this structure as well.

As can be seen from Figure 2, the dimer (and trimer and tetramer) ATDs are somewhat broader than the respective monomer ATDs and tail toward longer drift time. The tailing is a consequence of the exit ion funnel, i.e., an experimental artifact. The increased peak width might be a consequence that some of the higher energy isomers discussed above are present in small amounts. Furthermore, it should be noted that the calculated structures are (local) minima calculated at $0 \mathrm{~K}$, while the ion mobility experiments are performed at room temperature.

It is interesting to compare the dimer structures that we obtained for divalent metal centers with trivalent metal centers. In the case of $\mathrm{M}^{\mathrm{III}} \mathrm{TPPS}$ with $\mathrm{M}^{\mathrm{III}}=\mathrm{Fe}, \mathrm{Mn}$, we had previously observed $^{11,12}$ completely different structures for charge state 4and 5-: the monomeric units are not connected via the counterions but by bonds between the metal centers and the sulfonic acid groups. This can easily be understood electrostatically: since the metal centers replace two hydrogen atoms, the porphyrin center is essentially uncharged in the case of divalent metals, but carries a positive net charge for trivalent metals - which strongly attracts the negatively charged sulfonic acid groups.

Trimers. We obtained two charge states of the trimers in sufficient intensity to determine their collision cross sections: $\left[(\mathrm{MTPPS})_{3}+8 \mathrm{Na}\right]^{4-}$ and $\left[(\mathrm{MTPPS})_{3}+7 \mathrm{Na}\right]^{5-}$.

$\left[(\mathrm{MTPPS})_{3}+8 \mathrm{Na}\right]^{4-}$. For the quadruply charged trimer we measure a CCS of $477 \AA^{2}$ for $\mathrm{M}=\mathrm{Zn}, 479 \AA^{2}$ for $\mathrm{M}=\mathrm{Cu}$, and $484 \AA^{2}$ for $\mathrm{M}=\mathrm{Pd}$; again these are identical within the experimental uncertainty. It is interesting to compare these values with the CCS for the quintuply charged dimers (see above)-they are basically the same, although the trimers consists of 275 atoms instead of 181 atoms for the dimer. Obviously, the geometry of $\left[(\mathrm{MTPPS})_{3}+8 \mathrm{Na}\right]^{4-}$ must be very compact. Given available computational resources, the trimers are too large for DFT calculations with the TZVPP basis set (which is necessary to minimize basis set superposition errors, see above). Therefore, on the basis of our experience with the dimers we rely on PM7 to provide reasonable candidate structures for $\left[(\mathrm{ZnTPPS})_{3}+8 \mathrm{Na}\right]^{4-}$. The most favorable structure that we obtain is a face-to-face stack of the three porphyrins. The eight sodium counterions connect the three porphyrins via their sulfonic acid groups, four between each porphyrin pair (structure I, see Figure 6 and Supporting Information for a more complete list of candidate structures). This is also the most compact structure that we have found (and the most compact geometry that is physically reasonable). Its TM-CCS is $481 \AA^{2}$, again in perfect agreement (1\%) with the experimental value of $477 \AA^{2}$. The PA-CCS is again significantly smaller, $436 \AA^{2}$, implying that this method is not suitable for such large systems. Structure II represents an example of a distorted stack; its TM-CCS is $491 \AA^{2}$, slightly too large, and more than $1.5 \mathrm{eV}$ higher in energy. Planar side-byside structures (Figure 6, structure III) can be ruled out both on the of basis of their collision cross section (TM-CCS $691 \AA^{2}$, more than $200 \AA^{2}$ above the experimental value) as well as their relative energy $(1.7 \mathrm{eV}$ above structure $\mathrm{I})$. To summarize, it is clear that $\left[(\mathrm{MTPPS})_{3}+8 \mathrm{Na}\right]^{4-}$ consists of a an H-aggregatelike face-to-face stack of three porphyrins.

$\left[(\mathrm{MTPPS})_{3}+7 \mathrm{Na}\right]^{5-}$. Removing one sodium ion dramatically changes the collision cross section: for the quintuply charged trimers $\left[(\mathrm{MTPPS})_{3}+7 \mathrm{Na}\right]^{5-}$ we measure collision cross sections of $581 \AA^{2}$ for $\mathrm{M}=\mathrm{Zn}$ and $573 \AA^{2}$ for $\mathrm{M}=\mathrm{Pd}$. For $\mathrm{M}=$ $\mathrm{Cu}$ we identify two peaks at the limit of our experimental resolution, 563 and $587 \AA^{2}$. All of these collision cross sections are more than $100 \AA^{2}$ larger than what we obtain for the quadruply charged trimers. The lowest energy structure that we find (Figure 7, structure I) consists of a stack of two porphyrins connected with the third porphyrin by two sodium ions. Structure III represents a variant of this motif with one sodium atom at a different position. It is slightly higher in energy $(+0.34 \mathrm{eV})$. Both structures have similar cross sections, one slightly larger, the other slightly smaller than the experimental cross section. It is possible that we observe a mixture of both structures. A planar side-by-side geometry (structure II) is only $0.25 \mathrm{eV}$ higher in energy; so on the basis of energy it seems 


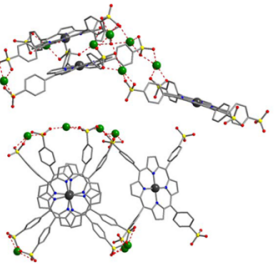

(I) $\triangle \mathrm{E}_{\mathrm{Pm} 7} \quad 0 \mathrm{eV}$ TM-CCS $567 \AA^{2}$ PA-CCS $532 \AA^{2}$

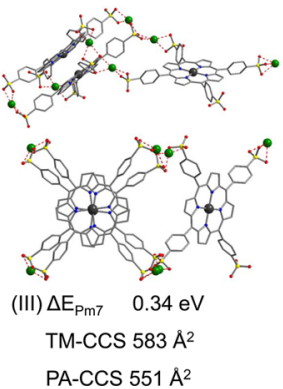

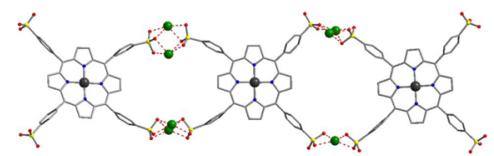

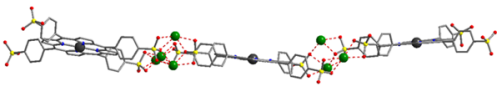

(II) $\Delta \mathrm{E}_{\mathrm{Pm} 7} \quad 0.25 \mathrm{eV}$ TM-CCS $691 \AA^{2}$ PA-CCS $674 \AA^{2}$
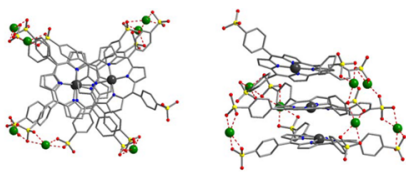

(IV) $\Delta \mathrm{E}_{\mathrm{Pm} 7} \quad 1.02 \mathrm{eV}$ TM-CCS $495 \AA^{2}$ PA-CCS $447 \AA^{2}$

Figure 7. Low-energy structures of $\left[(\mathrm{ZnTPPS})_{3}+7 \mathrm{Na}\right]^{5-}$ : relative energies and collision cross sections. The experimental collision cross section is $581 \AA^{2}$.

plausible that this isomer could be present in substantial amounts as well. However, on the basis of its cross section this can be ruled out. The TM-CCS of structure II is $691 \AA^{2}$, more than $100 \AA^{2}$ above the experimental value. The same holds true for the stack of three porphyrins (structure IV), the geometry favored for the lower charge state: it has a TM-CCS of $495 \AA^{2}$,

$80 \AA^{2}$ below the experimental value. Furthermore, it is significantly higher in energy $(+1.02 \mathrm{eV})$.

It is interesting to note that we do not observe any $\left[(\mathrm{ZnTPPS})_{3}+6 \mathrm{Na}\right]^{6-}$ : as can be seen from Figure 1 , at $m / z=$ 520 only $[\mathrm{ZnTPPS}+2 \mathrm{Na}]^{2-}$ and $\left[(\mathrm{ZnTPPS})_{2}+4 \mathrm{Na}\right]^{4-}$ are present. Apparently, six sodium cations cannot compensate the Coulomb repulsion of 12 negatively charged sulfonic acid groups. (For PdTPPS we see trace amounts of [(PdTPPS $)_{3}+$ $6 \mathrm{Na}]^{6-}$, see the corresponding mass spectrum in Supporting Information.)

As for the dimers we find completely different structural motifs for divalent versus trivalent metal centers: for the $\mathrm{M}^{\mathrm{III}}$ TPPS trimers $(\mathrm{M}=\mathrm{Mn}, \mathrm{Fe})$ at both charge state 5- and 6- we again find structures that are connected by bonds between sulfonic acid groups and central metal atoms rather than through the counterions (see refs 11 and 12; for a figure of the structures, see below).

Tetramers. $\left[(\mathrm{MTPPS})_{4}+10 \mathrm{Na}\right]^{6-}$. Among the tetramers, only $\left[(\mathrm{ZnTPPS})_{4}+10 \mathrm{Na}\right]^{6-},\left[(\mathrm{CuTPPS})_{4}+10 \mathrm{Na}\right]^{6-}$, and $\left[(\mathrm{PdTPPS})_{4}+10 \mathrm{Na}\right]^{6-}$ are present in sufficient intensity to determine a collision cross section. (Note that the [(MTPPS $)_{4}$ $+10 \mathrm{Na}]^{6-}$ signal corresponds to the small leading peak in the ATD of $m / z=701$. The main peak is [(MTPPS $\left.)_{2}+5 \mathrm{Na}\right]^{3-}$, see Figure 2 and Supporting Information.) We obtain cross sections of $661 \AA^{2}(\mathrm{Cu}, \mathrm{Zn})$ and $676 \AA^{2}(\mathrm{Pd})$ with an uncertainty of $5 \%$ due to the small signal intensities. As before, we perform the PM7 geometry optimizations with $\mathrm{Zn}$ as metal center. We find a structure consisting of a stack of three porphyrins with the fourth porphyrin connected in a side-byside fashion by two sodium ions to be lowest in energy (see Figure 8, structure I), a motif similar to what we find for $\left[(\mathrm{MTPPS})_{3}+7 \mathrm{Na}\right]^{5-}$. Its TM-CCS is $694 \AA^{2}$. Structure III
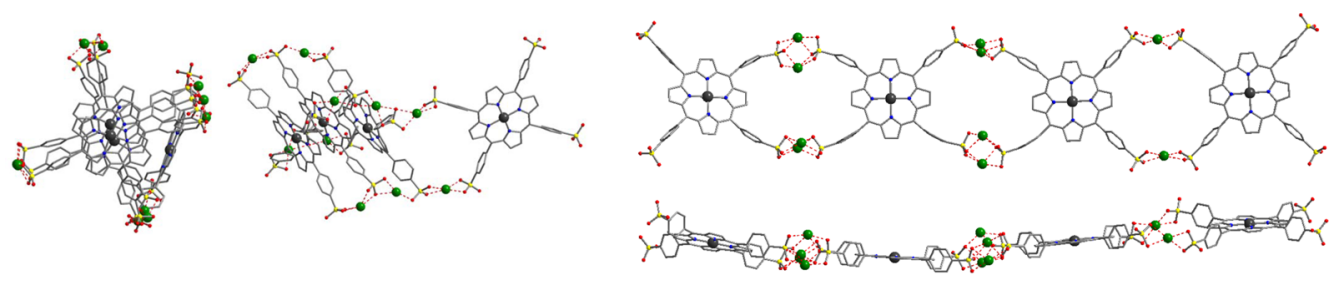

(I) $\Delta \mathrm{E}_{\mathrm{Pm} 7} \quad 0 \mathrm{eV}$ TM-CCS $694 \AA^{2}$ PA-CCS $634 \AA^{2}$

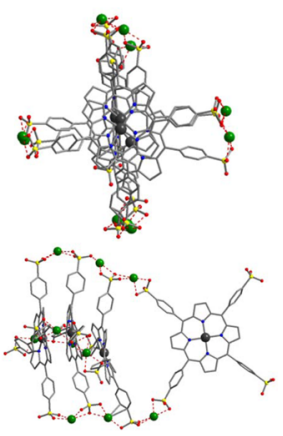

(III) $\Delta \mathrm{E}_{\mathrm{Pm} 7} \quad 0.69 \mathrm{eV}$ TM-CCS $672 \AA^{2}$ PA-CCS $610 \AA^{2}$
(II) $\Delta \mathrm{E}_{\mathrm{Pm} 7} \quad 0.17 \mathrm{eV}$ TM-CCS $914 \AA^{2}$ PA-CCS $887 \AA^{2}$
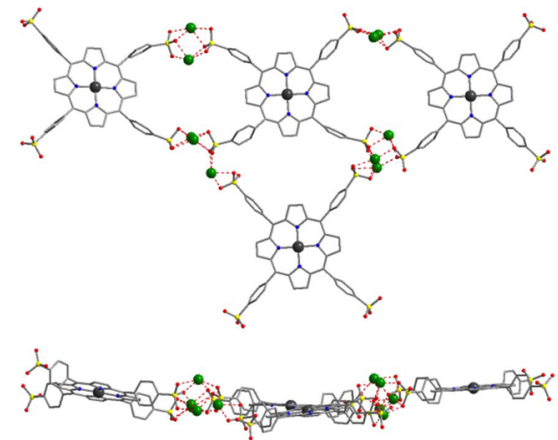

(IV) $\Delta \mathrm{E}_{\mathrm{Pm} 7} \quad 1.08 \mathrm{eV}$

TM-CCS $879 \AA^{2}$

PA-CCS $844 \AA^{2}$

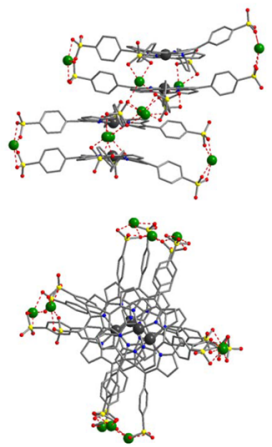

(V) $\triangle \mathrm{E}_{\mathrm{Pm} 7} \quad 1.34 \mathrm{eV}$

TM-CCS $597 \AA^{2}$

PA-CCS $525 \AA^{2}$

Figure 8. Low-energy structures of $\left[(\mathrm{ZnTPPS})_{4}+10 \mathrm{Na}\right]^{6-}$ : relative energies and collision cross sections. The experimental collision cross sections were determined only for the metal centers $\mathrm{Cu}$ and $\mathrm{Pd}$. They are 661 and $676 \AA^{2}$, respectively. 
$\left(+0.69 \mathrm{eV}, \mathrm{TM}-\mathrm{CCS} 672 \AA^{2}\right)$ represents a variant of the same motif. Both structures are in line with the experimental CCS. A planar side-by-side structure is energetically almost degenerate $(+0.17 \mathrm{eV}$, which is well within the uncertainty of the PM7 calculation), but its cross section (TM-CCS: $914 \AA^{2}$ ) is too large by more than $200 \AA^{2}$. Structure IV can be considered as another planar variant; it can be ruled out on the basis of its large TM-CCS (879 $\left.\AA^{2}\right)$ as well. A (slightly distorted) face-toface quadruple-decker stack (structure $\mathrm{V}$ ), on the other hand, has a TM-CCS of $597 \AA^{2}$, more than $60 \AA^{2}$ too low. All these structures can be ruled out.

\section{SUMMARY}

We have used electrospray ionization to prepare negatively charged oligomers of $\mathrm{M}^{\mathrm{II}}$ TPPS $\left(\mathrm{M}^{\mathrm{II}}=\mathrm{Zn}, \mathrm{Cu}, \mathrm{Pd}\right)$, in sizes up to tetramers and in different charge states (as determined by varying the number of their $\mathrm{Na}^{+}$counterions). The corresponding structures were then determined using a combination of ion mobility measurements and quantum chemical calculations. Under the fully isolated, gas-phase conditions of our experiment, we find that the structure of a given oligomer size and charge state is essentially independent of the central metal studied. Interestingly, oligomers in low negative charge states (which have a larger number of sodium counterions) tend to form stacked structures very similar to the $\mathrm{H}$-aggregate motif observed in solution. In contrast, for the higher negative charge states the stack opens as a consequence of the lower number of counterions that connect the TPPS units via Coulomb interactions with the negatively charged sulfonic acid groups. Note, however, that this holds true only for divalent metals as central atoms. For $\mathrm{M}^{\mathrm{III}} \mathrm{TPPS}$ with trivalent metal centers, $\mathrm{M}^{\mathrm{III}}=\mathrm{Fe}, \mathrm{Mn}$, we had previously observed $^{11,12}$ that the counterions only play a minor role. In this case, the ensuing daisy-chain-like structures are primarily determined by (partially ionic) bonds between the $\mathrm{M}^{\mathrm{III}}$ centers and the sulfonic acid groups. The structures obtained by quantum chemical calculations and confirmed by ion mobility spectrometry are summarized in Figure 9.

The results reported here directly impact our ongoing optical and photoelectron spectroscopic studies of the same metal-

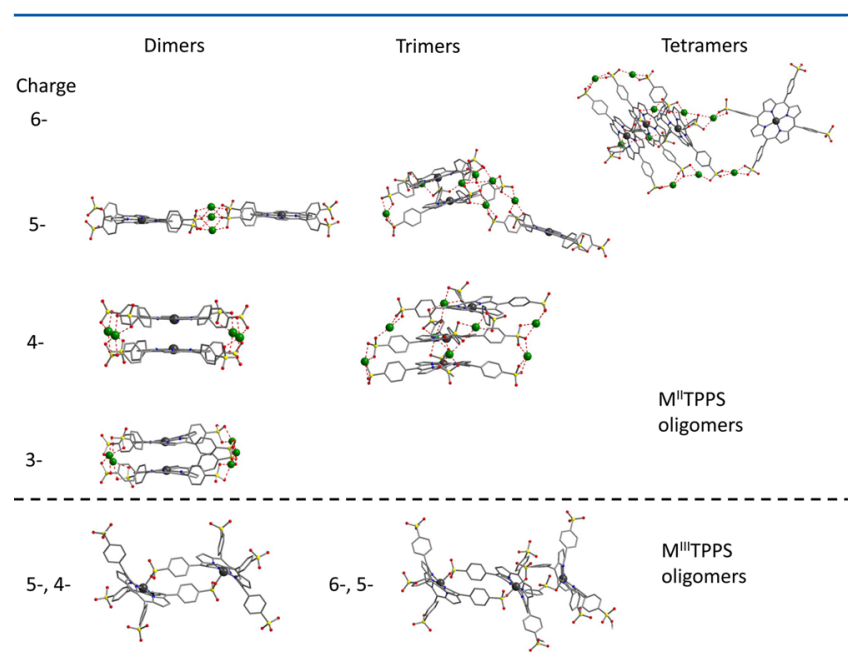

Figure 9. MTPPS oligomer structures as obtained by the combination of ion mobility spectrometry and quantum chemical calculations. Top: $\mathrm{M}^{\mathrm{II}} \mathrm{TPPS}, \mathrm{M}=\mathrm{Cu}, \mathrm{Zn}$, Pd; this work. Bottom: $\mathrm{M}^{\mathrm{III}} \mathrm{TPPS}, \mathrm{M}=\mathrm{Mn}, \mathrm{Fe}$, with protons as counterions; see refs 11 and 12 . loporphyrin oligomer systems. ${ }^{12,33}$ They demonstrate that much as in condensed phase chemical synthesis, ${ }^{34}$ gas-phase "Coulombic self-assembly" of metalloporphyrins allows one to controllably place (different) transition metal centers in a variety of different separations and relative orientations. This will allow systematic probes of how the positions of these metal centers influence their cooperative optical properties in the absence of a solvent (excited-state energies and dissipation channels).

A more technical aspect of this work pertains to the collision cross section calculation methodology. We find that the atomic parameters implemented in the MOBCAL program must be slightly adjusted in order to accurately describe the porphyrin oligomer multianions. For this we have used the monomers as calibration points. Under these conditions we can reproduce experimental CCS values using predicted minimum structures together with the trajectory method to within an accuracy of $2 \%$ (4\% for the tetramers). The much less sophisticated projection approximation (calibrated for the monomer as well) significantly underestimates the collision cross sections by up to $5 \%$ for the dimers and $8 \%$ for the trimers and tetramers.

\section{ASSOCIATED CONTENT}

\section{Supporting Information}

The Supporting Information is available free of charge on the ACS Publications website at DOI: 10.1021/acs.jpca.6b08062.

Typical mass spectra and arrival time distributions of CuTPPS and PdTPPS oligomers, PM7-optimized geometries (sorted with respect to their relative energy) of the ZnTPPS isomers discussed above as well as some high-energy isomers (PDF)

\section{AUTHOR INFORMATION}

\section{Corresponding Authors}

*E-mail: patrick.weis@kit.edu. Phone: +49 72160843306.

*E-mail: manfred.kappes@kit.edu. Phone: +49721 60842094.

Notes

The authors declare no competing financial interest.

\section{ACKNOWLEDGMENTS}

We thank the Deutsche Forschungsgemeinschaft (DFG) for funding through project $\mathrm{C} 6$ of the collaborative research centre SFB/TRR 88 "3MET" (Kooperative Effekte in Homo- und Heterometallischen Komplexen). Funding of an orbitrap mass spectrometer by DFG and Land Baden-Württemberg/KIT (Art $91 b)$ is also gratefully acknowledged.

\section{REFERENCES}

(1) The Porphyrin Handbook; Kadish, K. M., Smith, K. M., Guilard, R., Eds.; Academic Press: New York, 2003.

(2) Beletskaya, I.; Tyurin, V. S.; Tsivadze, A. Y.; Guilard, R.; Stern, C. Supramolecular Chemistry of Metalloporphyrins. Chem. Rev. 2009, 109, 1659-1713.

(3) Pasternack, R. F.; Gibbs, E. J.; Gaudemer, A.; Antebi, A.; Bassner, S.; DePoy, L.; Turner, D. H.; Williams, A.; Laplace, F.; Lansard, M. H.; et al. Molecular Complexes of Nucleosides and Nucleotides with a Monomeric Cationic Porphyrin and Some of Its Metal Derivatives. J. Am. Chem. Soc. 1985, 107, 8179-8186.

(4) Yushmanov, V. E.; Imasato, H.; Tominaga, T. T.; Tabak, M. H-1 NMR and Electronic Absorption Spectroscopy of Paramagnetic Water-soluble Meso-tetraarylsubstituted Cationic and Anionic Metalloporphyrins. J. Inorg. Biochem. 1996, 61, 233-250. 
(5) Gandini, S. C. M.; Yushmanov, V. E.; Tabak, M. Interaction of $\mathrm{Fe}(\mathrm{III})$ - and $\mathrm{Zn}$ (II)-tetra(4-sulfonatophenyl) Porphyrins with Ionic and Nonionic Surfactants: Aggregation and Binding. J. Inorg. Biochem. 2001, 85, 263-277.

(6) Ribó, J. M.; Crusats, J.; Farrera, J. A.; Valero, M. L. Aggregation in Water Solutions of Tetrasodium Diprotonated Meso-tetrakis(4sulfonatophenyl)porphyrin. J. Chem. Soc., Chem. Commun. 1994, 681-682.

(7) Ma, H.-L.; Jin, W.-J. Studies on the Effects of Metal Ions and Counter Anions on the Aggregate Behaviors of Meso-tetrakis(psulfonatophenyl) porphyrin by Absorption and Fluorescence Spectroscopy. Spectrochim. Acta, Part A 2008, 71, 153-160.

(8) Pasternack, R. F.; Schaefer, K. F.; Hambright, P. Resonance Light-scattering Studies of Porphyrin Diacid Aggregates. Inorg. Chem. 1994, 33, 2062-2065.

(9) Hollingsworth, J. V.; Richard, A. J.; Vicente, M. G. H.; Russo, P. S. Characterization of the Self-Assembly of meso-Tetra(4sulfonatophenyl)porphyrin (H2TPPS4-) in Aqueous Solutions. Biomacromolecules 2012, 13, 60-72.

(10) Micali, N.; Mallamace, F.; Romeo, A.; Purrello, R.; Monsù Scolaro, L. Mesoscopic Structure of meso-tetrakis(4-sulfonatophenyl)porphine J-aggregates. J. Phys. Chem. B 2000, 104, 5897-5904.

(11) Schwarz, U.; Vonderach, M.; Kappes, M.; Kelting, R.; Brendle, K.; Weis, P. Structural Characterization of Metalloporphyrin-oligomer Multianions by Mass Spectrometry and Ion Mobility SpectrometryObservation of Metastable Species. Int. J. Mass Spectrom. 2013, 339$340,24-33$.

(12) Schwarz, U.; Vonderach, M.; Armbruster, M. K.; Fink, K.; Kappes, M. M.; Weis, P. Cu(II)- and Mn(III)-Porphyrin-Derived Oligomeric Multianions: Structures and Photoelectron Spectra. J. Phys. Chem. A 2014, 118, 369-379.

(13) Bowers, M. T.; Kemper, P.R; von Helden, G.; van Koppen, P. A. M. Gas-Phase Ion Chromatography: Transition Metal State Selection and Carbon Cluster Formation. Science 1993, 260, 14461451.

(14) Clemmer, D. E.; Jarrold, M. F. Ion Mobility Measurements and their Applications to Clusters and Biomolecules. J. Mass Spectrom. 1997, 32, 577-592.

(15) Gidden, J.; Bushnell, J. E.; Bowers, M. T. Gas-phase Conformations and Folding Energetics of Oligonucleotides: dTG(-) and dGT(-). J. Am. Chem. Soc. 2001, 123, 5610-5611.

(16) Wyttenbach, T.; von Helden, G.; Bowers, M. T. Gas-phase Conformation of Biological Molecules: Bradykinin. J. Am. Chem. Soc. 1996, 118, 8355-8364.

(17) Henderson, S. C.; Valentine, S. J.; Counterman, A. E.; Clemmer, D. E. ESI/Ion Trap/Ion Mobility/Time-of-flight Mass Spectrometry for Rapid and Sensitive Analysis of Biomolecular Mixtures. Anal. Chem. 1999, 71, 291-301.

(18) Jarrold, M. F. Helices and Sheets in Vacuo. Phys. Chem. Chem. Phys. 2007, 9, 1659-1671.

(19) Baker, E. S.; Bushnell, J. E.; Wecksler, S. R.; Lim, M. D.; Manard, M. J.; Dupuis, N. F.; Ford, P. C.; Bowers, M. T. Probing Shapes of Bichromophoric Metal-organic Complexes using Ion Mobility Mass Spectrometry. J. Am. Chem. Soc. 2005, 127, 1822218228.

(20) Vonderach, M.; Ehrler, O. T.; Weis, P.; Kappes, M. M. Combining Ion Mobility Spectrometry, Mass Spectrometry, and Photoelectron Spectroscopy in a High-Transmission Instrument. Anal. Chem. 2011, 83, 1108-1115.

(21) Mason, E. A.; McDaniel, E. W. Transport Properties of Ions in Gases; Wiley: New York, 1988.

(22) Stewart, J. J. P. MOPAC 2012; Stewart Computational Chemistry: Colorado Springs, CO, 2012; http://OpenMOPAC.net.

(23) Furche, F.; Ahlrichs, R.; Hättig, C.; Klopper, W.; Sierka, M.; Weigend, F. Turbomole. WIREs Comput. Mol. Sci. 2014, 4, 91-100.

(24) Becke, A. D. Density-functional Exchange-energy Approximation with Correct Asymptotic-behavior. Phys. Rev. A: At., Mol., Opt. Phys. 1988, 38, 3098-3100.
(25) Perdew, J. P. Density-functional Approximation for the Correlation-energy of the Inhomogeneous Electron-gas. Phys. Rev. B: Condens. Matter Mater. Phys. 1986, 33, 8822-8824.

(26) Weigend, F.; Ahlrichs, R. Balanced Basis Sets of Split Valence, Triple Zeta Valence and Quadruple Zeta Valence Quality for H to Rn: Design and Assessment of Accuracy. Phys. Chem. Chem. Phys. 2005, 7, $3297-3305$

(27) von Helden, G.; Hsu, M.-T.; Gotts, N.; Bowers, M. T. Carbon Cluster Cations with up to 84 Atoms: Structures, Formation Mechanism, and Reactivity. J. Phys. Chem. 1993, 97, 8182-8192.

(28) Mesleh, M. F.; Hunter, J. M.; Shvartsburg, A. A.; Schatz, G. C.; Jarrold, M. F. Structural Information from Ion Mobility Measurements: Effects of the Long-range Potential. J. Phys. Chem. 1996, 100, $16082-16086$.

(29) Shvartsburg, A. A.; Jarrold, M. F. An Exact Hard-spheres Scattering Model for the Mobilities of Polyatomic Ions. Chem. Phys. Lett. 1996, 261, 86-91.

(30) Fleischer, E. B.; Miller, C. K.; Webb, L. E. Crystal and Molecular Structures of Some Metal Tetraphenylporphines. J. Am. Chem. Soc. 1964, 86, 2342-2347.

(31) Scheidt, W. R.; Kastner, M. E.; Hatano, K. Stereochemistry of the Toluene Solvate of Alpha, Beta, Gamma, Deltatetraphenylporphinatozinc(II). Inorg. Chem. 1978, 17, 706-710.

(32) Faure, S.; Stern, C.; Espinosa, E.; Douville, J.; Guilard, R.; Harvey, P. D. Triplet-triplet Energy Transfer Controlled by the Donor-acceptor Distance in Rigidly Held Palladium-containing Cofacial Pisporphyrins. Chem. - Eur. J. 2005, 11, 3469-3481.

(33) Jäger, P.; Brendle, K.; Schwarz, U.; Himmelsbach, M.; Armbruster, M. K.; Fink, K.; Weis, P.; Kappes, M. M. Q and Soret Band Photoexcitation of Isolated Palladium Porphyrin Tetraanions Leads to Delayed Emission of Nonthermal Electrons over Microsecond Time Scales. J. Phys. Chem. Lett. 2016, 7, 1167-1172.

(34) Harvey, P.; Stern, C.; Gros, C.; Guilard, R. The Photophysics and Photochemistry of Cofacial Free Base and Metallated Bisporphyrins held Together by Covalent Architectures. Coord. Chem. Rev. 2007, 251, 401-428. 